ISSN 0103-9954

\title{
PRIMERO RELATO DE Costalimaita ferruginea (Fabricius, 1801) (COLEOPTERA: CHRYSOMELIDAE) EM EUCALIPTO NO ESTADO DO MATO GROSSO DO SUL
}

\author{
FIRST RECORD OF Costalimaita ferruginea (Fabricius, 1801) (COLEOPTERA: \\ CHRYSOMELIDAE) IN EUCALYPTUS IN MATO GROSSO DO SUL STATE
}

\author{
Samir Oliveira Kassab ${ }^{1}$ Thiago Alexandre Mota ${ }^{2}$ \\ Fabrício Fagundes Pereira ${ }^{3}$ Paulo Rogério Beltramin da Fonseca ${ }^{4}$
}

\begin{abstract}
RESUMO
Relatou-se a ocorrência do besouro amarelo do eucalipto, Costalimaita ferruginea (Fabricius, 1801) (Coleoptera: Chrysomelidae), em novembro de 2009, em plantios clonais de Eucalyptus grandis x Eucalyptus urophylla, no município de Três Lagoas, Mato Grosso do Sul. Este é o primeiro registro dessa espécie em mudas clonais de eucalipto no Estado. No Brasil, Costalimaita ferruginea é considerada uma das principais pragas do eucalipto, devido a sua capacidade de causar injúrias, como o desfolhamento nas partes apicais da planta, promovendo perdas significativas no desenvolvimento e inviabilizando a comercialização da madeira e seus derivados.
\end{abstract}

Palavras-chave: besouro amarelo do eucalipto; entomologia florestal; mudas clonais; insetos-praga.

\begin{abstract}
The occurrence of the eucalyptus yellow beetle Costalimaita ferruginea (Fabricius, 1801) (Coleoptera: Chrysomelidae) was repoted in November 2009 in clonal plantations of Eucalyptus grandis x Eucalyptus urophylla in Três Lagoas, Mato Grosso do Sul state. This is the first record of this species of eucalyptus seedlings in this state. In Brazil, Costalimaita ferruginea is considered a major pest of eucalyptus because of its ability to cause injuries such as the apical defoliation in parts of the plant, promoting significant losses in the development and inhibiting the marketing of wood and its derivatives.
\end{abstract}

Keywords: eucalyptus yellow beetle; forest entomology; clone seedling; pest-insects.

\section{INTRODUÇÃO}

A cultura do eucalipto tornou-se uma importante atividade econômica no Brasil, ocupando cerca de 5,8 milhões de hectares (VALVERDE, 2007), devido ao fato desta espécie possuir rápido crescimento, capacidade produtiva e adaptabilidade em diversos ambientes, e, sobretudo, a sua diversidade de espécies, o que torna possível atender demanda de grande parte dos segmentos que utilizam produtos florestais. A implantação das monoculturas como maciços florestais homogêneos, favorece a ocorrência de altas populações de insetospraga, devido à disponibilidade de alimento e a simplificação do ambiente, o que pode prejudicar o empreendimento florestal (OHRMART e EDWARDS, 1991).

1. Biólogo, Doutorando do Programa de Pós-graduação em Entomologia e Conservação da Biodiversidade, Universidade Federal da Grande Dourados. Caixa Postal 533, CEP 79804-970, Dourados (MS). samirkassab@gmail.com

2. Biólogo, Doutorando do Programa de Pós-graduação em Entomologia e Conservação da Biodiversidade, Universidade Federal da Grande Dourados. Caixa Postal 533, CEP 79804-970, Dourados (MS). thiamota@hotmail.com

3. Engenheiro Agrônomo, Dr., Professor da Faculdade de Ciências Biológicas e Ambientais, Universidade Federal da Grande Dourados. Caixa Postal 533, CEP 79804-970, Dourados (MS). fabriciofagundes@ufgd.edu.br

4. Engenheiro Agrônomo, Doutorando do Programa de Pós-graduação em Agronomia, Universidade Federal da Grande Dourados. Caixa Postal 533, CEP 79804-970, Dourados (MS).prbeltramin@hotmail.com

Recebido para publicação em 4/03/2010 e aceito em 25/11/2010 
Relatos de coleópteros de importância econômica em áreas florestais no Mato Grosso do Sul são escassos. As espécies de besouros da madeira, Euplatypus parallelus, Euplatypus sp. (Coleoptera: Euplatypodidae) e Xyleborus affinis (Coleoptera: Scolytidae) foram registrados atacando florestas de Pinus sp. no município de Ribas do Rio Pardo (ZANUNCIO et al., 2002).

O besouro desfolhador do eucalipto, Costalimaita ferruginea (Fabricius, 1801) (Coleoptera: Chrysomelidae), popularmente conhecido como "besouro amarelo" destaca-se como uma das principais pragas dessa cultura e, devido ao aumento das áreas de plantio, surtos desses insetos, tornam-se cada vez mais frequentes com prejuízos expressivos.

Em média, as fêmeas de Costalimaita ferruginea ovipositam 90 ovos, os quais apresentam coloração amarela e brilhante com um período embrionário de 8 dias. As larvas desse inseto vivem no solo e alimentam-se de raízes de gramíneas. No período larval, são conhecidos até o presente momento, dois estádios de desenvolvimento. O primeiro ínstar apresenta aproximadamente 19 dias e o segundo cerca de 40 dias e após a ocorrência das primeiras chuvas os adultos emergem do solo (GOULD et al., 2002).

Costalimaita ferruginea tem preferência por brotos e partes apicais da planta de eucalipto, o que pode causar perdas significativas do parênquima clorofiliano, prejudicando assim seu desenvolvimento (MENDES et al., 1998). A desfolha do terço superior da copa, juntamente com o ponteiro principal cortado por este inseto pode implicar em perdas de até $55,80 \mathrm{~m}^{3} / \mathrm{ha}$, o que equivale a $\mathrm{R} \$ 2.232,00 / \mathrm{ha}$ (MENDES, 2004).

No intuito de auxiliar no mapeamento da distribuição geográfica do inseto, o presente trabalho objetivou relatar a ocorrência do "besouro amarelo" em plantios com mudas clonais de Eucalyptus grandis x Eucalyptus urophylla, localizados no município de Três Lagoas, Estado de Mato Grosso do Sul.

\section{MATERIAL E MÉTODO}

Em novembro de 2009, em uma área de plantio comercial, foi observada a presença de um besouro amarelo em mudas clonais de Eucalyptus grandis $\mathrm{x}$ Eucalyptus urophylla, no município de Três Lagoas, MS, cuja latitude é $20^{\circ} 45^{\prime} 04^{\prime \prime} \mathrm{S}$, longitude de $51^{\circ} 40^{\prime} 42^{\prime}$ 'W, e altitude de $319 \mathrm{~m}$. A área em que foi registrada a presença do inseto era de 135 ha. O espaçamento do plantio de $3 \times 2 \mathrm{~m}$, com plantas de 18 meses de idade. Os exemplares coletados machos e fêmeas foram enviados ao Prof. Dr. Norivaldo dos Anjos do Departamento de Biologia Animal da Universidade Federal de Viçosa, para identificação.

\section{RESULTADOS E DISCUSSÃO}

$\mathrm{O}$ inseto foi identificado como sendo Costalimaita ferruginea (Fabricius, 1801) (Coleoptera: Chrysomelidae), sendo este o primeiro registro dessa espécie em mudas clonais do híbrido de eucalipto Eucalyptus grandis $x$ Eucalyptus urophylla no Estado de Mato Grosso do Sul. Os exemplares coletados apresentam coloração amarelada (Figura 1A), as plantas atacadas tinham as folhas perfuradas (Figura 1B) e algumas exibiam sinais de ramificação intensa (Figura 1C).

Resultados de estudos recentes evidenciam que, no Brasil, Costalimaita ferruginea, apresenta-se estabelecido nas regiões Sul, Sudeste, Nordeste e Centro-Oeste, comumente encontrado em áreas cultivadas com eucalipto próximas às pastagens, pois sua fase larval ocorre no solo, onde se alimentam das raízes de gramíneas. No Brasil, há relatos da espécie nos Estados de São Paulo, Paraná, Goiás, Rio Grande do Norte, Rio Grande do Sul, Pará, Maranhão, Bahia, Espírito Santo e Minas Gerais (COSTA et al., 2008).

$\mathrm{Da}$ área total de plantio de eucalipto observada (78.000 ha), foi verificada a distribuição generalizada do inseto em 135 ha (Figura 1D). Uma hipótese que pode justificar a ocorrência do surto nos talhões de eucalipto é a substituição da cobertura nativa por extensas áreas florestais, fato esse que favorece o desenvolvimento deste inseto, devido à abundância de alimento. Além disso, as condições climáticas da região podem favorecer seu ciclo biológico, diminuindo o período de tempo necessário para o desenvolvimento de uma geração, resultando na explosão populacional do inseto (ELEK et al., 2000). É importante ressaltar ainda que, informalmente sabe-se que produtos de largo espectro de ação e não registrados para a cultura do eucalipto são utilizados para o controle de insetos, sendo um agravante por ser uma possível causa da mortalidade dos inimigos naturais de Costalimaita ferruginea. 

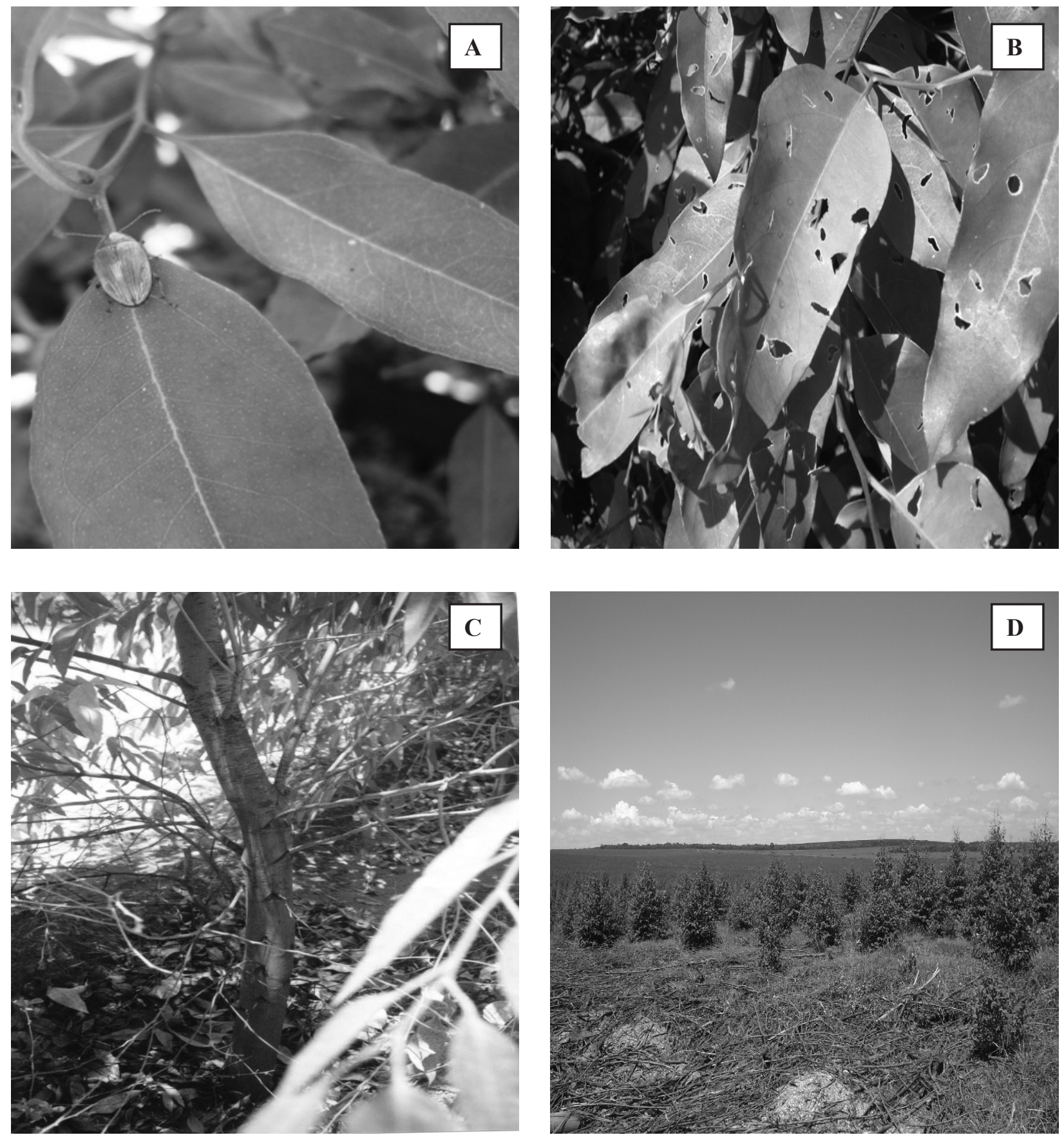

FIGURA 1: Adulto de Costalimaita ferruginea (Coleoptera: Chrysomelidae) (A); Folhas de Eucalyptus grandis x Eucalyptus urophylla com danos (B); Ramificação intensa na planta (C); Área de eucalipto atacada pelo besouro amarelo (D).

FIGURE 1: Adult of Costalimaita ferruginea (Coleoptera: Chrysomelidae) (A); Leaves of Eucalyptus grandis $\mathrm{x}$ Eucalyptus urophylla with damage (B); Intense branching plant (C). Area eucalyptus attacked by yellow beetle (D). 


\section{CONCLUSÕES}

Como esteé o primeiro registro da ocorrência do besouro amarelo do eucalipto Costalimaita ferruginea em plantios clonais de Eucalyptus grandis $x$ Eucalyptus urophylla no Mato Grosso do Sul, Brasil, recomenda-se o monitoramento da população desse inseto para precaver-se de prejuízos com eventuais surtos que possam ocorrer.

\section{AGRADECIMENTOS}

Ao Prof. Dr. Norivaldo dos Anjos (UFV), pela identificação do inseto e à Empresa Fibria, por possibilitar a visita técnica aos estudantes do Programa de Pós-Graduação em Entomologia e Conservação da Biodiversidade da UFGD.

\section{REFERÊNCIAS BIBLIOGRÁFICAS}

COSTA, E. C. et. al. Entomologia Florestal. Santa Maria: UFSM, 2008. $240 \mathrm{p}$.

ELEK, J.; BASHFORD, R.; CANDY, S; Manual for managing leaf beetle defoliation in eucalypt plantations. Australia: Forestry Tasmania, 2000. $53 \mathrm{p}$.
MENDES, J. P. E. Efeito do ataque do Costalimaita ferruginea (Fabr.) (Coleoptera: Chrysomelidae) sobre o crescimento e produção de Eucalyptus grandis W. Hill ex Maiden. 2004. 49 f. Tese (Doutorado em Entomologia) - Departamento de Biologia Animal, Universidade Federal de Viçosa, Viçosa, 2004.

MENDES, J. P. E. et al. Monitoramento do besouro amarelo. Folha Florestal, n. 91, p. 8-9, 1998.

GOULD, W. P.; RAGA, A. Pests of guava. In: Tropical fruit pests and pollinators: biology, economic importance, natural enemies and control., New York: CABI Publishing, 2002. p. 295-313.

OHRMART, C. P.; EDWARDS, P. B. Insect herbivory on eucalyptus. Annual Review of Entomology, Durham, v. 36, p. 637-657, 1991.

VALVERDE, S. R. Biotecnologia florestal como diferencial da competitividade Brasileira. In: OLIVEIRA, A.B. (Org.). Biotecnologia florestal. Viçosa: UFV, 2007. v.1, p.363 - 374.

ZANUNCIO, J. C. et al. Occurrence of Euplatypus parallelus, Euplatypus sp. (Col: Euplatypodidae) and Xyleborus affinis (Col: Scolytidae) in Pinus sp. in Ribas do Rio Pardo, Mato Grosso do Sul, Brasil. Revista Árvore, Viçosa, v. 26, n. 3, p. 387-389, maio/jun. 2002. 